\title{
AN ABSTRACT DELAY-DIFFERENTIAL EQUATION MODELLING SIZE DEPENDENT CELL GROWTH AND DIVISION*
}

\author{
M. GYLLENBERG ${ }^{\dagger}$ AND H. J. A. M. HEIJMANS ${ }^{\ddagger}$
}

\begin{abstract}
A two-phase model for the growth of a single cell population structured by size is formulated and analysed. The model takes the form of a delay-differential equation in a Banach space. Using positivity arguments, we describe the spectrum of the infinitesimal generator of the semigroup associated with solutions. Under a certain condition on the growth rate of individual cells the semigroup is compact after finite time. This enables us to determine the ultimate behavior of solutions and prove the existence of a stable size distribution.
\end{abstract}

Key words. structured populations, cell cycle, first order partial differential equation with delay and transformed argument, delay-differential equation in a Banach space, strongly continuous semigroup, generation expansion, positive operator, Riesz operator, spectral theory, stable size distribution

AMS(MOS) subject classifications. Primary 34K30, 92A15, 35R10

1. Introduction. In this paper we study a mathematical model for the dynamics of a population of single cells which can be distinguished from each other according to their size and the particular cell cycle phase they are in. Models for populations of dividing organisms incorporating size or age-size structure have been formulated, among others, by Bell and Anderson (1967) and Sinko and Streifer (1971) and have recently been investigated by Diekmann, Heijmans and Thieme (1984) and Heijmans (to appear $\mathrm{a}, \mathrm{b}$ ). We refer to the book of Metz and Diekmann (to appear) for a general exposition of the dynamics of physiologically structured populations. There exists a vast literature on models for progress through the cell cycle and its various phases, see for instance the book of Eisen (1979). Tyson and Hannsgen (1984) and Hannsgen and Tyson (1984) have studied cell cycle models which also take the cell size distribution into account.

We consider a model in which we assume that the cell cycle consists of two distinct phases. The first phase is of variable length. The cells in this phase cannot divide-they increase in size and, provided they do not die, they will eventually enter the second phase. This second phase, which is assumed to have constant duration, can be considered as an idealization of the mitotic period. At the conclusion of this phase cells split into two equal parts and the newborn daughter cells start the cycle in the first phase. It is assumed that the cells in the first phase are fully characterized by their size. By this we mean that for instance the growth, death and transition rates are functions of size, and of size only. Moreover, we assume that in the second phase the growth and death rates are functions of size, but the fission rate is a (delta-) function of the time elapsed since entering the second phase.

Our model could be considered as a generalization of the one-phase model studied by Diekmann et al. (1984) since if one formally puts the duration of the second phase equal to zero our fundamental equation (2.1) reduces to the corresponding equation in the above-mentioned paper.

\footnotetext{
* Received by the editors April 22, 1985; accepted for publication October 1, 1985.

${ }^{\dagger}$ Helsinki University of Technology, SF-02150 Espoo 15, Finland.

₹Centre for Mathematics and Computer Science, Kruislaan 413, 1098 SJ Amsterdam, The Netherlands.
} 
The model could easily be modified (without essentially affecting the results) to allow for more complicated cell cycles and asymmetric division, see Gyllenberg (to appear).

Diekmann et al. (1984) showed that under reasonable hypotheses the population will ultimately grow or decay exponentially and they gave conditions on the individual growth rate under which the size distribution converges towards a so-called stable size distribution. In this paper we shall prove that the two-phase model exhibits a similar asymptotic behavior, if we adapt the condition on the growth rate.

It turns out to be mathematically convenient to write the model as a delay-differential equation in a Banach space. Our main tools will be the theory of strongly continuous semigroups and spectral theory, in particular of positive operators.

The organization of the paper is as follows. In $\$ 2$ we write down the balance equation for the size distribution of the population of the first phase. This equation, which is a first-order hyperbolic PDE with time delay, transformed argument and singular coefficients, is then reformulated as an abstract linear delay-differential equation in a Banach space. In $\$ 3$ we prove well-posedness of the abstract problem and associate a strongly continuous semigroup of bounded linear operators with the solution. In $\S 4$ we represent the solution as a generation expansion and give conditions under which the semigroup is compact after finite time. In $\$ 5$ we study the related eigenvalue problem and characterize the spectrum of the infinitesimal generator of the semigroup. In $\$ 6$ we use the results of the preceding sections to state and prove the main result on the asymptotic behavior of solutions.

2. The model. The starting point of our investigation is the balance equation for the size distribution of cells in the first phase

$$
\begin{aligned}
& \frac{\partial}{\partial t} n(t, x)+\frac{\partial}{\partial x}(g(x) n(t, x)) \\
& \quad=-\mu(x) n(t, x)-b(x) n(t, x)+\frac{2 p\left(y^{-1}(x)\right) b\left(y^{-1}(x)\right)}{y^{\prime}\left(y^{-1}(x)\right)} n\left(t-r, y^{-1}(x)\right) .
\end{aligned}
$$

Here $t$ denotes time and $x$ denotes size. The unknown $n$ is the size distribution of cells in the first phase, i.e. the integral $\int_{x_{1}}^{x_{2}} n(t, x) d x$ represents the number of cells in the first phase with size between $x_{1}$ and $x_{2}$ at time $t$. The functions $g, \mu$ and $b$ (which are assumed to be known) are the rates at which cells of size $x$ grow, die and transit to the second phase, respectively. $r>0$ is the constant duration of the second phase, $y(x)$ is the size of a newborn cell whose mother entered the second phase (exactly $r$ time units before) with size $x$ and $p(x)$ is the fraction of cells who survive the second phase given that they entered it with size $x$.

The left-hand side of (2.1) is the derivative along characteristic curves and describes an individual's motion in the time-size continuum due to growth. The first term on the right-hand side describes the loss due to deaths and the second the loss due to transition to the second phase. The last term describes the birth of cells from mother cells completing their second phase: of those cells that entered the second phase $r$ time units ago with size $y^{-1}(x)$ a fraction $p\left(y^{-1}(x)\right)$ will successfully complete the phase and give rise to two new cells of size $x$. The factor $1 / y^{\prime}\left(y^{-1}(x)\right)$ may seem strange. It is due to the fact that cells giving birth to daughters in the size interval $(x, x+d x)$ left the first phase with size in the interval

$$
\left(y^{-1}(x), y^{-1}(x)+\frac{d x}{y^{\prime}\left(y^{-1}(x)\right)}\right) .
$$


If one formally puts $r=0$, one should take $y(x)=\frac{1}{2} x$ and the last term in (2.1) reduces to the corresponding term in the model of Diekmann et al. (1984), namely $4 b(2 x) n(t, 2 x)$.

We assume that cells cannot enter the second phase before they have reached a minimal size $x_{0}$ and that $y\left(x_{0}\right) \leqq x_{0}$. It follows that cells with size less than $\alpha:=y\left(x_{0}\right)$ cannot exist. This fact is expressed by the boundary condition

$$
n(t, \alpha)=0
$$

which supplements (2.1).

We assume further that each cell in the first phase must either die or transit to the second phase before it reaches a maximal size (normalized to $x=1$ ) of the first phase. This requires that the integral $\int_{\alpha}^{x}[b(s) / g(s)] d s$ diverges as $x \uparrow 1$ and that the source term in (2.1) is interpreted as zero for $x \geqq \beta:=y(1)$ where $\beta$ is assumed to be smaller than 1 . We once and for all make the convention that all functions containing $y^{-1}(x)$ as an argument are given the value zero for $x \geqq \beta$. The possible sizes a cell in the first phase can have thus lie in the interval $(\alpha, 1)$, which should be chosen as the domain of $x$ in (2.1). In order to obtain a well-posed problem, an initial function $\nu$ should be prescribed on $[-r, 0] \times[\alpha, 1]$ :

$$
n(t, x)=\nu(t, x), \quad-r \leqq t \leqq 0, \quad \alpha \leqq x<1 .
$$

Concerning the growth, death and transition rates and the other given functions, we assume (compare Diekmann et al. (1984)):

$$
\begin{aligned}
& \left(H_{y}\right) y \in C^{1}\left[x_{0}, 1\right], y^{\prime}>0, \alpha:=y\left(x_{0}\right) \leqq x_{0} \text { and } \beta:=y(1)<1 . \\
& \left(H_{p}\right) p \in C\left[x_{0}, 1\right], 0<p(x) \leqq 1, x \in\left[x_{0}, 1\right] . \\
& \left(H_{g}\right) g \in C[\alpha, 1], g(x)>0, x \in[\alpha, 1] . \\
& \left(H_{\mu}\right) \mu \in C[\alpha, 1], \mu(x) \geqq 0, x \in[\alpha, 1] . \\
& \left(H_{b}\right) b \in C[\alpha, 1], b(x)=0, x \in\left[\alpha, x_{0}\right], b(x)>0, x \in\left(x_{0}, 1\right), \\
& \quad \lim _{x \uparrow 1} \int_{x_{0}}^{x} b(s) d s=\infty \text { and } \frac{b(x)}{g(x)} E(x) \leqq M<\infty, \quad x \in[\alpha, 1] .
\end{aligned}
$$

In the last condition we have used the notation

$$
E(x)=\exp \left(-\int_{\alpha}^{x} \frac{b(s)+\mu(s)}{g(s)} d s\right) .
$$

$E(x) / E(y)$ is the probability that a cell of size $y$ remains in the first phase at least until it reaches size $x$ and $\int_{x_{1}}^{x_{2}}(b(s) / g(s)) E(s) d s$ is the probability that a cell with size $x_{0}$ enters the second phase when its size is between $x_{1}$ and $x_{2}$. By our assumptions the (possibly defective) probability density $b E / g$ is not only an $L^{1}$-function but also bounded and continuous.

We point out that some of the assumptions could be weakened at the cost of some minor technical difficulties. For instance, if $y\left(x_{0}\right)>x_{0}$ we could redefine $x_{0}$ in the following way. Let $x_{n}=y\left(x_{n-1}\right), n=1,2, \cdots$. Since $y(1)<1, x_{n} \rightarrow x_{0}^{\prime}$ as $n \rightarrow \infty$ where $x_{0}^{\prime}$ is the smallest fixed point of $y$. $x_{0}^{\prime}$ could then be taken as the new $x_{0}$. A similar procedure has been carried out in Heijmans (to appear, b). Guided by Diekmann et al. (1984) we substitute

$$
n(t, x)=\frac{E(x)}{g(x)} u(t, x)
$$


into (2.1) and obtain

$$
\frac{\partial u}{\partial t}(t, x)+g(x) \frac{\partial u}{\partial x}(t, x)=k(x) u\left(t-r, y^{-1}(x)\right),
$$

where

$$
k(x)=\left\{\begin{array}{l}
2 \frac{g(x) p\left(y^{-1}(x)\right) b\left(y^{-1}(x)\right)}{E(x) y^{\prime}\left(y^{-1}(x)\right) g\left(y^{-1}(x)\right)} E\left(y^{-1}(x)\right), \quad x \in[\alpha, \beta), \\
0, \quad x \in(\beta, 1) .
\end{array}\right.
$$

The boundary condition (2.2) becomes

$$
u(t, \alpha)=0
$$

and the initial condition (2.3) changes into

$$
u(t, x)=\phi(t, x), \quad t \in[-r, 0], \quad x \in[\alpha, 1],
$$

where

$$
\phi(t, x)=[g(x) / E(x)] \nu(t, x) .
$$

We shall look for solutions which are continuous functions of $t$ with values in the Banach space $X=L^{1}[\alpha, 1]$. Therefore we rewrite the problem (2.6), (2.8), (2.9) as the following abstract delay equation:

$$
\begin{aligned}
& \frac{d u(t)}{d t}=B u(t)+L u(t-r), \quad t>0, \\
& u(t)=\phi(t), \quad t \in[-r, 0] .
\end{aligned}
$$

Here $B$ is the unbounded closed linear operator defined by $B \psi=-g \psi^{\prime}$ for all $\psi$ in the domain $\mathscr{D}(B)=\{\psi \in X \mid \psi$ is absolutely continuous on $[\alpha, 1], \psi(\alpha)=0\}$ and $L$ is the operator defined for all $\psi$ in $X$ by $(L \psi)(x)=k(x) \psi\left(y^{-1}(x)\right)$. It follows from $\left(H_{b}\right)$ that $L$ is a bounded linear operator on $X$. $\phi$ is a given initial function in

$$
C=C([-r, 0] ; X) \text {. }
$$

The rest of the paper is devoted to the investigation of so-called mild solutions of the abstract problem (2.10)-(2.11).

3. Existence and uniqueness and the corresponding semigroup. It is obvious that the operator $B$ defined at the end of $\S 2$ generates a strongly continuous semigroup $\{S(t)\}_{t \geqq 0}$ of linear operators on $X$. In fact, let

$$
G(x)=\int_{\alpha}^{x} \frac{d \xi}{g(\xi)}
$$

and define

$$
X(t, x)=G^{-1}(G(x)+t), \quad 0 \leqq G(x)+t \leqq G(1) .
$$

(Note that $G^{-1}$ is well defined on $[0, G(1)]$ because $g>0$.) Then $S(t)$ is given for every $\psi \in X$, every $t \geqq 0$ and almost every $x \in[\alpha, 1]$ by

$$
(S(t) \psi)(x)= \begin{cases}\psi(X(-t, x)) & \text { if } G(x)-t>0 \\ 0 & \text { if } G(x)-t \leqq 0\end{cases}
$$


Note that $S(t)=0$ for $t \geqq G(1)$. Observe that $X(t, x)$ is the solution of the initial value problem

$$
\frac{d X}{d t}=g(X), \quad X(0, x)=x
$$

and hence $X(t, x)$ represents the size of a cell at time $t$ which had size $x$ at time zero.

If there exists a continuously differentiable function $u$ satisfying (2.10), (2.11), it satisfies the following integral equation (variation of constants formula)

$$
u(t)=S(t) \phi(0)+\int_{0}^{t} S(t-s) L u(s-r) d s
$$

for $t>0$. Any continuous function $u$ which satisfies (3.5), (2.11) is called a mild solution of the initial value problem.

Travis and Webb (1974) have investigated existence, uniqueness and semigroup properties of a class of functional differential equations in Banach spaces. Some of their basic results can be applied to the present problem. As a special case of Proposition 2.1 of Travis and Webb (1974) we have

Proposition 3.1. For each $\phi \in C$ there exits a unique mild solution $u(\phi)$ : $[-r, \infty) \rightarrow X$ of the initial value problem $(2.10),(2.11)$.

If $u$ is a continuous function $[-r, \infty) \rightarrow X$ we denote by $u_{s}(s \geqq 0)$ the element of $C$ defined by

$$
u_{s}(\theta)=u(s+\theta), \quad \theta \in[-r, 0] .
$$

For each $t \geqq 0$ we define $T(t): C \rightarrow C$ by $T(t) \phi=u(\phi)_{t}, \phi \in C$, where $u(\phi)$ is the unique mild solution of (2.10), (2.11) given by Proposition 3.1. The results of Travis and Webb (1974, Prop. 3.1.) give us the following:

PROPOSITION 3.2. $\{T(t)\}_{t \geqq 0}$ is a strongly continuous semigroup of linear operators on $C$. The infinitesimal generator $A$ of $\{T(t)\}_{t \geqq 0}$ is given by

$$
\begin{aligned}
& \mathscr{D}(A)=\left\{\phi \in C \mid \phi^{\prime} \in C, \phi(0) \in \mathscr{D}(B), \phi^{\prime}(0-)=B \phi(0)+L \phi(-r)\right\}, \\
& (A \phi)(\theta)=\phi^{\prime}(\theta), \quad \theta \in[-r, 0] .
\end{aligned}
$$

One of our main objectives is to describe the large time behavior of mild solutions. Such information can be obtained from spectral properties of $T(t)$. If the semigroup is compact after finite time, then by a well-known spectral mapping theorem (cf. Pazy (1983, Chap. 2)) the spectrum of $T(t)$ is completely determined by the spectrum of its infinitesimal generator $A$. In the next section we give conditions under which $T(t)$ is indeed compact for $t$ large enough and in $\S 5$ we use positivity arguments to give a rather precise characterization of the spectrum of $A$. It turns out that the same condition which ensures compactness of $T(t)$ guarantees the existence of a strictly dominant real eigenvalue of $A$. A combination of these results enables us to determine the asymptotic behavior of solutions.

4. Generation expansion and compactness of the semigroup. In the theory of linear autonomous differential-delay equations in finite-dimensional Euclidean spaces $\mathbb{R}^{n}$ the semigroup associated with the solution acts on the space $C\left([-r, 0] ; \mathbb{R}^{n}\right)$ and it is a relatively easy consequence of Ascoli's theorem that the semigroup is compact for $t \geqq r$ (cf. Hale (1977, Chap. 7)). In our case $\mathbb{R}^{n}$ is replaced by the infinite-dimensional Banach space $X$ and the proof of Lemma 1.1 of Hale (1977, Chap. 7) does not carry over to this case, simply because the Heine-Borel theorem fails in infinite-dimensional 
spaces. Neither can we use the compactness results of Travis and Webb (1974) since their results depend heavily on the assumption that the operator $B$ generates a semigroup which which is compact for all $t>0$ and this condition is not satisfied in the problem under consideration.

In order to prove compactness of the semigroup corresponding to a related problem (without delay), Diekmann et al. (1984) wrote down a generation expansion for the solution. Here we shall use similar methods. The larger dimensionality of our state space makes the compactness proof a bit more involved than in the above-mentioned paper.

In the problem under consideration where we have to take account of individuals present at negative time, it makes sense to define also the -1 st generation. We write

$$
u(t ; \phi)=\sum_{i=-1}^{\infty} u^{i}(t ; \phi),
$$

where

$$
\begin{aligned}
& u^{-1}(t ; \phi)= \begin{cases}\phi(t), & -r \leqq t \leqq 0, \\
0, & t>0,\end{cases} \\
& u^{0}(t ; \phi)=S(t) \phi(0)+\int_{0}^{t} S(t-\tau) L u^{-1}(\tau-r ; \phi) d \tau, \quad t \geqq 0
\end{aligned}
$$

and the higher generations are obtained by iteration of the integral operator.

$$
u^{i+1}(t ; \phi)=\int_{0}^{t} S(t-\tau) L u^{i}(\tau-r ; \phi) d \tau, \quad t \geqq 0, \quad i \geqq 0 .
$$

Let for $i \geqq 0$ and $t \in[r, \infty)$ the operator family $T^{i}(t): C \rightarrow C$ be defined by

$$
T^{i}(t) \phi=u^{i}(\phi)_{t}
$$

Now let $i \geqq 0, t \geqq r$ and $\theta \in[-r, 0]$, then

$$
\begin{aligned}
\left(T^{i+1}(t) \phi\right)(\theta) & =u^{i+1}(t+\theta ; \phi)=\int_{0}^{t+\theta} S(t+\theta-\tau) L u^{i}(\tau-r ; \phi) d \tau \\
& =\int_{-\theta}^{t} S(t-s) L u^{i}(s+\theta-r ; \phi) d s \\
& =\int_{-\theta}^{t} S(t-s) L\left(T^{i}(s-r) \phi\right)(\theta) d s \\
& =\int_{r}^{t} S(t-s) L\left(T^{i}(s-r) \phi\right)(\theta) d s .
\end{aligned}
$$

For a bounded operator $F: X \rightarrow X$ we define the bounded operator $\tilde{F}: C \rightarrow C$ by $(\tilde{F} \phi)(\theta)=F(\phi(\theta))$ for all $\phi \in C$. Thus we can write

$$
T^{i+1}(t)=\int_{r}^{t} \tilde{S}(t-s) \tilde{L} T^{i}(s-r) d s, \quad t \geqq r, \quad i \geqq 0 .
$$

We note that $T(t)=\sum_{i=0}^{\infty} T^{i}(t), t \geqq r$. If we can prove that $T^{1}(t)$ is compact for $t \geqq r$ then it follows from (4.6) that $T^{i+1}(t)$ is compact for $t \geqq r$ and $i \geqq 1$ and this finally yields that $T(t)$ is compact, $t \geqq r+G(1)$, since $T^{0}(t)=0$ if $t \geqq r+G(1)$. Therefore the rest of this section is concerned with a proof of the compactness of $T^{1}(t), t \geqq r$.

The following version of Ascoli's theorem can be found in Martin (1976, Thm. II, 3.2.). 
LEMMA 4.1. $A$ set $V$ in $C$ is precompact if the following conditions are satisfied:

i) $V$ is bounded.

ii) The family $V$ is equicontinuous.

iii) For each $\theta \in[-r, 0]$ the subset $\{\phi(\theta) \mid \phi \in V\}$ of $X$ is precompact.

An easy calculation shows that

(4.7) $u^{0}(t, x ; \phi)=\phi(0, X(-t, x))+\int_{0}^{t} k(X(-\tau, x)) \phi\left(t-\tau-r, y^{-1}(X(-\tau, x))\right) d \tau$,

(4.8) $u^{1}(t, x ; \phi)=\int_{0}^{t} k(X(-\tau, x))\left\{\phi\left(0, X\left(-t+\tau+r, y^{-1}(X(-\tau, x))\right)\right)\right.$

$$
\begin{gathered}
+\int_{0}^{t-\tau-r} k\left(X\left(-\sigma, y^{-1}(X(-\tau, x))\right)\right) \\
\left.\cdot \phi\left(t-\sigma-\tau-2 r, y^{-1}\left(X\left(-\sigma, y^{-1}(X(-\tau, x))\right)\right)\right) d \sigma\right\} d \tau .
\end{gathered}
$$

In trying to prove compactness of $T^{1}(t)$ it becomes clear that we need some relation between $g$ and $y$. We shall make the following assumption:

Assumption 4.2.

$$
g(x) y^{\prime}(x)<g(y(x)), \quad x_{0} \leqq x \leqq 1 .
$$

Below we shall give an interpretation of this inequality.

THEOREM 4.3. If Assumption 4.2 is satisfied, then the semigroup $T(t)$ is compact for $t \geqq r+G(1)$.

Proof. We have already explained that it suffices to show that $T^{1}(t)$ is compact for $t \geqq r$. Instead of (4.8) we can write

$$
u^{1}(t, x ; \phi)=u_{1}^{1}(t, x ; \phi)+u_{2}^{1}(t, x ; \phi),
$$

where

$$
\begin{array}{r}
u_{1}^{1}(t, x ; \phi)=\int_{0}^{t} k(X(-\tau, x)) \cdot \phi\left(0, X\left(-t+\tau+r, y^{-1}(X(-\tau, x))\right)\right) d \tau \\
u_{2}^{1}(t, x ; \phi)=\int_{0}^{t} k(X(-\tau, x))\left\{\int_{-r}^{t-\tau-2 r} k\left(X\left(s-t+\tau+2 r, y^{-1}(X(-\tau, x))\right)\right)\right. \\
\left.\cdot \phi\left(s, y^{-1}\left(X\left(s-t+\tau+2 r, y^{-1}(X(-\tau, x))\right)\right)\right) d s\right\} d \tau
\end{array}
$$

where in this second expression we have substituted

$$
s=t-\sigma-\tau-2 r .
$$

Let $T_{j}^{1}(t): C \rightarrow C$ for $t \geqq r, j=1,2$ be given by

$$
\left(T_{j}^{1}(t) \phi\right)(\theta)=u_{j}^{1}(t+\theta ; \phi), \quad \theta \in[-r, 0] .
$$

Here we shall prove that $T_{2}^{1}(t)$ is compact for $t \geqq r$. The easier proof of compactness of $T_{1}^{1}(t), t \geqq r$ is omitted.

Let $t \geqq r$ be fixed and let for $R>0$ the subset $C_{R}$ of $C$ be given by $C_{R}=\{\phi \in$ $\left.C \mid\|\phi\|_{C} \leqq R\right\}$. We will show that $V=\left\{T_{2}^{1}(t) \phi \mid \phi \in C_{R}\right\}$ obeys the conditions of Lemma 4.1. Obviously $V$ is bounded. Now we replace $\tau$ by the variable

$$
z=y^{-1}\left(X\left(s-t+\tau+2 r, y^{-1}(X(-\tau, x))\right)\right) \text {. }
$$


Then

$$
X(-s+t-\tau-2 r, y(z))=y^{-1}(X(-\tau, x))=\xi .
$$

Differentiation with respect to $\tau$ yields:

$$
-g(\xi)+\frac{g(\xi)}{g(y(z))} \cdot y^{\prime}(z) \frac{d z}{d \tau}=-\frac{g(y(\xi))}{y^{\prime}(\xi)} .
$$

Therefore, if Assumption 4.2 is satisfied, $d z / d \tau$ never becomes zero and replacing $\tau$ by $z$ in the expression of $u_{2}^{1}$ one obtains

$$
u_{2}^{1}(t, x ; \phi)=\iint_{\Omega(t, x)} Q(s, z ; t, x) \phi(s, z) d s d z,
$$

where $\iint_{\Omega(t, x)} d s d z$ is uniformly continuous in $t$ and $x$ in bounded subsets of the $(t, x)$-plane and $Q(s, z ; t, x)$ is uniformly continuous in $s, z, t$ and $x$ in bounded subsets of the $(s, z, t, x)$-plane. At this point the reader will have no difficulty in seeing that $V$ indeed obeys conditions (ii) and (iii) of Lemma 4.1.

In the case where there is no delay, which has been studied by Diekmann et al. (1984), the function $y$ is given by $y(x)=\frac{1}{2} x$ and Assumption 4.2 reduces to $\frac{1}{2} g(x)<$ $g\left(\frac{1}{2} x\right), x_{0} \leqq x \leqq 1$, and this is indeed the condition imposed in that paper in order to establish compactness of the semigroup.

To see the biological meaning of Assumption 4.2, consider two identical cells in the first phase with size $x>x_{0}$. Assume that one of the cells immediately enters the second phase. It will divide after $r$ time units. Assume further that the two daughter cells will remain in the first phase for $t$ time units. $t+r$ time units after our initial moment each daughter cell will have size $X(t, y(x))$. The other cell is assumed to behave differently. It first grows for $t$ time units reaching size $X(t, x)$, then enters the second phase and finally at time $t+r$ divides into two daughter cells of size $y(X(t, x))$ each. Assumption 4.2 guarantees that

$$
y(X(t, x))<X(t, y(x)) .
$$

This can be seen as follows. Differentiation of $G(x)-G(y(x))$ shows that this expression is increasing in $x$ if Assumption 4.2 is satisfied. Now for $t>0$ and $x, \alpha \leqq x \leqq$ $X(-t, 1)$ we have that $x<X(t, x)$ and therefore

$$
G(x)-G(y(x))<G(X(t, x))-G(y(X(t, x)))=t+G(x)-G(y(X(t, x))) ;
$$

hence

$$
G(y(X(t, x)))<t+G(y(x))
$$

which implies (4.9). This thought experiment shows that the combination of growth and division provides a dispersion mechanism for cell size, which is essential for proving compactness and also, as we shall see in the following section, for proving some sort of strong positivity.

If $\beta<x_{0}$, then every cell has to pass size $x_{0}$ in each cycle. If Assumption 4.2 fails for all $x \in\left[x_{0}, 1\right]$ (which corresponds to the case where individual cells grow exponentially throughout the cell cycle), then $\tau:=G(x)-G\left(x_{0}\right)+r+G\left(x_{0}\right)-G(y(x))$ is constant. But $\tau$ is the time elapsed between the event when the mother cell passes size $x_{0}$ and the event when the two daughter cells pass size $x_{0}$. Thus $\tau$ can be considered as the effective cycle time. In the case of exponential individual growth the cycle time $\tau$ is the same for all cells; it does not depend on size; there is no dispersion. 
Finally we point out that Assumption 4.2 implies

$$
y(x)<x \text { for all } x \in\left(x_{0}, 1\right)
$$

which is a strengthening of $\left(H_{y}\right)$. To see this, let $x \in\left(x_{0}, 1\right)$ and take $t>0$ such that $X\left(t, x_{0}\right)=x$. Then (4.9) implies

$$
y(x)=y\left(X\left(t, x_{0}\right)\right)<X\left(t, y\left(x_{0}\right)\right) \leqq X\left(t, x_{0}\right)=x .
$$

5. The spectrum of $\boldsymbol{A}$. In this section we combine ideas similar to those of Travis and Webb (1974), Hale (1977, Chap. 7) and Heijmans (to appear, a) to describe the spectrum of the generator $A$ and, in particular, to prove the existence of a strictly dominant, algebraically simple real eigenvalue. Assumption 4.2 is not presupposed unless this is explicitly stated.

Let us first introduce some notation. The norm of a Banach space $Z$ is denoted by $\|\cdot\|_{Z} \cdot Z^{*}$ stands for the dual space of $Z$. We let $\langle\Phi, \phi\rangle_{Z}$ be the duality pairing of $\phi \in Z$, $\Phi \in Z^{*}$. For an operator $T$ defined on a domain $\mathscr{D}(T) \subset Z$ with values in $Z$ we let $\sigma(T), P \sigma(T)$ and $\rho(T)$ denote the spectrum, point spectrum and resolvent set of $T$ respectively. $r(T)$ is the spectral radius, $\mathscr{N}(T)$ the kernel and $\mathscr{R}(T)$ the range of $T$.

By definition, $\lambda \in \rho(A)$ if and only if the equation

$$
(\lambda I-A) \phi=\psi
$$

has a unique solution $\phi \in \mathscr{D}(A)$ for all $\psi$ in $C$ and $\phi$ depends continuously on $\psi$. By Proposition 3.2 each $\phi$ in $\mathscr{D}(A)$ is continuously differentiable on $[-r, 0]$ and $A \phi=\phi^{\prime}$. Hence (5.1) can be rewritten as

$$
\lambda \phi(\theta)-\phi^{\prime}(\theta)=\psi(\theta), \quad \theta \in[-r, 0]
$$

and it follows that every solution $\phi$ of (5.2) is given by

$$
\phi(\theta)=e^{\lambda \theta} \phi(0)+\int_{\theta}^{0} e^{\lambda(\theta-s)} \psi(s) d s, \quad \theta \in[-r, 0] .
$$

In particular,

$$
\phi(-r)=e^{-\lambda r_{\phi}}(0)+\int_{-r}^{0} e^{-\lambda(r+s)} \psi(s) d s .
$$

On the other hand, Proposition 3.2 also tells us that

$$
\phi^{\prime}(0)=B \phi(0)+L \phi(-r)
$$

for all $\phi \in \mathscr{D}(A)$. Combining (5.2), (5.4) and (5.5), one obtains

$$
\Delta(\lambda) \phi(0)=\psi(0)+H(\lambda) \psi,
$$

where for each $\lambda \in \mathbb{C}$ the operator $\Delta(\lambda)$ with domain $\mathscr{D}(\Delta(\lambda))=\mathscr{D}(B)$ and values in $X$ is defined by

$$
\Delta(\lambda)=\lambda I-B-e^{-\lambda r} L
$$


and $H(\lambda)$ is defined on all of $C$ by

$$
H(\lambda) \psi=L\left(\int_{-r}^{0} e^{-\lambda(r+s)} \psi(s) d s\right), \quad \psi \in C .
$$

We can now prove the following.

Proposition 5.1. (a) $\lambda \in \sigma(A)$ if and only if $0 \in \sigma(\Delta(\lambda))$.

(b) $\lambda \in \operatorname{P\sigma }(A)$ if and only if $0 \in \operatorname{P\sigma }(\Delta(\lambda))$. Moreover, $\operatorname{dim} \mathscr{N}(\lambda I-A)=$ $\operatorname{dim} \mathscr{N}(\Delta(\lambda))$.

Proof. (a) Above we have shown that if $\phi \in \mathscr{D}(A)$ is a solution of (5.1), then $\phi(0)$ satisfies (5.6). Conversely, if $\phi(0) \in X$ satisfies (5.6) then the function $\phi$ given by (5.3) belongs to $\mathscr{D}(A)$ and is a solution of (5.1). To complete the proof of (a), it suffices to show that the right-hand side of (5.6) covers $X$ as $\psi$ ranges over $C$. In order to see this, consider $\psi \in C$ given by $\psi(s)=f(s) w$ where $w \in X$ and the scalar function $f$ defined on $[-r, 0]$ satisfies (i) $f(0)=1$, (ii) $\int_{-r}^{0} e^{-\lambda s} f(s) d s=0$. It is obvious that $\psi(0)+H(\lambda) \psi=w$.

b) Suppose $\lambda \in P \sigma(A)$ and let $\phi \in C, \phi \neq 0$ satisfy $A \phi=\lambda \phi$. Then $\phi(\theta)=\phi(0) e^{\lambda \theta}$ and $\Delta(\lambda) \phi(0)=0$. From $\phi \neq 0$ it follows that $\phi(0) \neq 0$ and therefore $0 \in \operatorname{P\sigma }(\Delta(\lambda))$. Similarly, $0 \in P \sigma(\Delta(\lambda) \Rightarrow \lambda \in P \sigma(A)$. The second relation follows immediately.

Proposition 5.1 characterizes the spectrum and the point spectrum of $A$ acting in the space $C=C([-r, 0], X)$ in terms of the operator $\Delta(\lambda)$ acting in the simpler space $X$. Below we shall investigate the spectral properties of $\Delta(\lambda)$ with the aid of yet another operator and eventually obtain a rather precise description of $\sigma(A)$.

Consider the equation

$$
\Delta(\lambda) w=f
$$

that is,

$$
\lambda w(x)+g(x) w^{\prime}(x)-e^{-\lambda r} k(x) w\left(y^{-1}(x)\right)=f(x)
$$

where $f \in X$. We are looking for solution $w \in \mathscr{D}(\Delta(\lambda))=\mathscr{D}(B)$. Following Heijmans (to appear, a), we transform (5.10) into an integral equation by means of the following substitution:

$$
w(x)=e^{-\lambda G(x)} v(x)
$$

Then (5.10) takes the form

$$
v^{\prime}(x)-k_{\lambda}(x) v\left(y^{-1}(x)\right)=\frac{f(x)}{g(x)} e^{\lambda G(x)},
$$

where by definition

$$
k_{\lambda}(x)= \begin{cases}\frac{k(x)}{g(x)} e^{-\lambda\left[G\left(y^{-1}(x)\right)-G(x)+r\right]}, & x \in[\alpha, \beta), \\ 0, & x \in[\beta, 1) .\end{cases}
$$

Since $w$ as a member of $\mathscr{D}(B)$ should be continuous and vanish at $x=\alpha$ the same must be true for $v$. We therefore look for solutions $v \in Y$ of (5.12) where $Y$ is the Banach space

$$
Y=\{v \in C[\alpha, 1] \mid v(\alpha)=0\} .
$$

Integration of (5.12) yields

$$
v-K(\lambda) v=U(\lambda) f
$$


where for $\lambda \in \mathbb{C}$ the operators $K(\lambda): Y \rightarrow Y$ and $U(\lambda): X \rightarrow X$ are defined by

$$
\begin{array}{cc}
{[K(\lambda) v](x)=\int_{\alpha}^{(x, \beta)^{-}} k_{\lambda}(\xi) v\left(y^{-1}(\xi)\right) d \xi,} & v \in Y, \quad x \in[\alpha, 1], \\
{[U(\lambda) f](x)=\int_{\alpha}^{x} \frac{f(\xi)}{g(\xi)} e^{\lambda G(\xi)} d \xi,} & f \in X .
\end{array}
$$

The advantage of the formulation using the operators $K(\lambda)$ and $U(\lambda)$ is that these are compact (the proof of this fact is standard) and that $K(\lambda)$ has useful positivity properties. Observe that the range of $U(\lambda)$ lies in $Y$.

We can now prove the following theorem concerning some relations between the spectra of $A, \Delta(\lambda)$ and $K(\lambda)$.

THEOREM 5.2. The following conditions are equivalent.

(a) $\lambda \in \sigma(A)$.

(b) $\lambda \in P \sigma(A)$.

(c) $0 \in \sigma(\Delta(\lambda))$.

(d) $0 \in P \sigma(\Delta(\lambda))$.

(e) $1 \in \sigma(K(\lambda))$.

(f) $1 \in P \sigma(K(\lambda))$.

Moreover, if $K(\lambda) v=v$ for some $\lambda \in \mathbb{C}$ and $v \in Y$, then $w$ given by (5.11) belongs to $\mathscr{D}(B)$ and satisfies $\Delta(\lambda) w=0$. If $0 \notin \sigma(\Delta(\lambda))$, then $\Delta(\lambda)^{-1}$ is compact.

Proof. In Proposition 5.1 we have already proved (a) $\Leftrightarrow$ (c) and (b) $\Leftrightarrow$ (d).

Putting $f=0$ one observes by comparing (5.9) and (5.15) that (d) $\Leftrightarrow$ (f) and that the eigenvector $v$ belonging to the eigenvalue 1 of $K(\lambda)$ corresponds to the eigenvector $w$ belonging to the eigenvalue 0 of $\Delta(\lambda)$.

(e) $\Leftrightarrow$ (f) follows directly from the compactness of $K(\lambda)$.

Since trivially $(\mathrm{b}) \Rightarrow(\mathrm{a})$ it remains to show that $(\mathrm{c}) \Rightarrow(\mathrm{e})$. To this end, suppose that $1 \notin \sigma(K(\lambda))$ which means that $I-K(\lambda)$ is invertible. For each $f \in X$ there exists therefore a unique solution $v \in Y$ of (5.15). But then $w$ defined by (5.11) satisfies (5.9). Hence $0 \in \sigma(\Delta(\lambda))$.

To prove compactness of $\Delta(\lambda)^{-1}$ for $0 \in \rho(\Delta(\lambda))$ observe that since $U(\lambda)$ is compact and $(I-K(\lambda))^{-1}$ is bounded, the mapping $f \rightarrow v$ defined by (5.15) is compact. The transformation $v \rightarrow w$ defined by (5.11) is obviously bounded, hence $\Delta(\lambda)^{-1}: f \rightarrow w$ is compact.

One important consequence of Theorem 5.2 is that the spectrum of $A$ consists solely of eigenvalues $((a) \Leftrightarrow(b))$. We emphasize that in order to establish this result we have not used compactness of $T(t)$, which would also imply the equivalence of (a) and (b).

Theorem 5.2 gives two entirely different characterizations of $\sigma(A)$-one in terms of $\Delta(\lambda)$, the other in terms of $K(\lambda)$. These characterizations will also be used for different purposes in the analysis to follow. $\Delta(\lambda)$ will prove to be of great importance in determining the algebraic and analytic properties of the eigenvalues and the resolvent operator of $A . K(\lambda)$ turns out to play a fundamental role in the investigation of the location of the eigenvalues in the complex plane.

We start by writing down an explicit expression for the resolvent operator $R(\lambda, A)$ : $C \rightarrow C$ of $A$. It follows from (5.3) and (5.6) that for $\lambda \in \rho(A)(\neq \varnothing$, since by a standard result for semigroups $\lambda \in \rho(A)$ for all $\lambda$ with $\operatorname{Re} \lambda$ large enough)

$$
(R(\lambda, A) \psi)(\theta)=e^{\lambda \theta}\left\{\Delta(\lambda)^{-1}(\psi(0)+H(\lambda) \psi)+\int_{\theta}^{0} e^{-\lambda s} \psi(s) d s\right\} .
$$


Hence $R(\lambda, A)=R_{1}(\lambda)+R_{2}(\lambda)$, where for $\lambda \in \rho(A)$, the bounded operators $R_{1}(\lambda)$ and $R_{2}(\lambda)$ are given by

$$
\begin{aligned}
& \left(R_{1}(\lambda) \psi\right)(\theta)=e^{\lambda \theta} \cdot \Delta(\lambda)^{-1}(\psi(0)+H(\lambda) \psi), \quad \psi \in C, \\
& \left(R_{2}(\lambda) \psi\right)(\theta)=\int_{\theta}^{0} e^{\lambda(\theta-s)} \psi(s) d s, \quad \psi \in C .
\end{aligned}
$$

From the boundedness of $H(\lambda): C \rightarrow X$ and the compactness of $\Delta(\lambda)^{-1}$ if $\lambda \in \rho(A)$ it follows that $R_{1}(\lambda): C \rightarrow C$ is compact if $\lambda \in \rho(A)$. Furthermore $R_{2}(\lambda)$ is quasinilpotent, i.e. $r\left(R_{2}(\lambda)\right)=0$ if $\lambda \in \mathbb{C}$. This can be shown as follows. Define the norm $\|\cdot\|_{\gamma}$ on $C$ as follows: $\|\psi\|_{\gamma}=\sup _{-r \leqq \theta \leqq 0}\left\|e^{-\gamma \theta} \psi(\theta)\right\|_{X}$. (This norm is equivalent to the original norm $\|\cdot\|_{C}$.) Now let $\gamma \in \mathbb{R}$ be such that $\gamma+\operatorname{Re} \lambda>0$. A straightforward calculation shows that $\left\|R_{2}(\lambda) \psi\right\|_{\gamma} \leqq(1 /(\gamma+\operatorname{Re} \lambda))\|\psi\|_{\gamma}$. Therefore $r\left(R_{2}(\lambda)\right) \leqq 1 /(\gamma+\operatorname{Re} \lambda)$ for all $\gamma>-\operatorname{Re} \lambda$ and this yields the result. As a sum of a compact and a quasinilpotent operator $R(\lambda, A)$ is a Riesz operator (cf. Dowson (1978)). The following result was proved by Lay (1970, Thm. 4.6).

THEOREM 5.3. Let $Z$ be an infinite-dimensional Banach space and let $T$ be a closed operator on $Z$ with nonempty resolvent set. Suppose that there exists an $\alpha \in \rho(T)$ such that $R(\alpha, T)$ is a Riesz operator. Then $\sigma(T)$ is a countable set of poles of $R(\lambda, T)$ of finite rank with $\infty$ the only possible point of accumulation.

As a consequence we have the following result.

COROllary 5.4. If $\lambda_{0} \in \sigma(A)$ then $\lambda_{0}$ is a pole of $R(\lambda, A)$ with residue of finite rank.

Remark 5.5. (a) For all $\lambda_{0} \in \sigma(A)$ we have that $\lambda_{0}$ is a pole of order $p$ of $(\Delta(\lambda))^{-1}$ iff $\lambda_{0}$ is a pole of order $p$ of $R(\lambda, A)$. This follows easily from (5.18) and the fact that $e^{\lambda \theta}$, the operator $H(\lambda)$ and the operator from $C$ to $X$ given by $\psi \rightarrow \int_{\theta}^{0} e^{-\lambda s} \psi(s) d s$ define entire functions.

(b) If Assumption 4.2 is satisfied, then the semigroup $T(t)$ is compact after finite time, and therefore the Browder essential spectrum (see e.g. Webb (1985) for a definition) $\sigma_{\text {ess }}(T(t))=\{0\}, t>0$, and now Corollary 5.4 follows immediately from Proposition 4.13 of Webb (1985) which says among other things

$$
\left\{e^{\lambda t} \mid \lambda \in \sigma_{\text {ess }}(A)\right\} \subset \sigma_{\text {ess }}(T(t)), \quad t>0 .
$$

The operator $K(\lambda)$ is very similar to an operator studied by Heijmans (to appear, a). Using essentially the same methods, based on the positivity of $K(\lambda)$ for $\lambda \in \mathbb{R}$, one can prove the following result. For readers consulting the above mentioned reference we mention that $K(\lambda)$ corresponds to $T_{\lambda}$ and that $x_{0}>\alpha$ and $x_{0}=\alpha$ respectively correspond to the cases $a>0$ and $a=0$ of that paper.

LEMMA 5.6. There exists $a \lambda_{d} \in \mathbb{R}$ such that

i) 1 is an algebraically simple eigenvalue of $K\left(\lambda_{d}\right)$.

ii) The associated eigenvector $v_{d} \in Y$ is strictly positive on $(\alpha, 1]$.

iii) All elements $\lambda \in \sigma(A)$ satisfy $\operatorname{Re} \lambda \leqq \lambda_{d}$.

Let $X_{+}$be the subset of $X$ consisting of all functions which are nonnegative a.e.; then $X_{+}$defines a cone in $X$ and with the induced ordering $X$ is a Banach lattice (see e.g. Schaefer (1974)). Define $C_{+}$as

$$
C_{+}=\left\{\phi \in C \mid \phi(\theta) \in X_{+}, \theta \in[-r, 0]\right\} .
$$

With the ordering induced by the cone $C_{+}$the space $C$ becomes a Banach lattice as well. 
Now we can prove the following important result.

THEOREM 5.7. The eigenvalue $\lambda_{d}$ of $A$ is algebraically simple. The eigenvector $\phi_{d}$ satisfies $\phi_{d}(\theta, x)=e^{\lambda_{d} \theta} w_{d}(x)$ where $w_{d} \in Y$ and $w_{d}(x)>0, x \in(\alpha, 1]$. The dual eigenvector $\Phi_{d}$, determined by $A^{*} \Phi_{d}=\lambda_{d} \Phi_{d}$ is strictly positive, i.e. $\phi \in C_{+}, \phi \neq 0$ implies that $\left\langle\Phi_{d}, \phi\right\rangle_{c}>0$.

Proof. As in Theorem 5.4 of Heijmans (to appear, a) we can show that $\lambda_{d}$ is a simple pole of $\Delta(\lambda)^{-1}$ and that the eigenvalue 0 of $\Delta\left(\lambda_{d}\right)$ has geometric multiplicity one. Combined with Proposition 5.1(b) and Remark 5.5(a) this yields the algebraic simplicity of the eigenvalue $\lambda_{d}$ of $A$. Let $v_{d}$ be given by Lemma 5.6 and $w_{d}$ by (5.11); then $\Delta\left(\lambda_{d}\right) w_{d}=0$. Now $\phi_{d} \in C_{+}$given by $\phi_{d}(\theta)=e^{\lambda_{d} \theta} w_{d}$ satisfies indeed the conditions stated in the theorem.

An easy calculation shows that $R(\lambda, A)$ defines a positive operator with respect to the cone $C_{+}$if $\lambda>\lambda_{d}$. Now let $\lambda_{0}>\lambda_{d}$ be fixed. Then $r\left(R\left(\lambda_{0}, A\right)\right)=1 /\left(\lambda_{0}-\lambda_{d}\right)$ and a standard result from positive operator theory says that $R\left(\lambda_{0}, A\right) * \Phi_{d}=\left(1 /\left(\lambda_{0}-\lambda_{d}\right)\right) \Phi_{d}$ for some positive functional $\Phi_{d} \neq 0$. Since $R\left(\lambda_{0}, A\right)^{*}=R\left(\lambda_{0}, A^{*}\right)$ (cf. Taylor and Lay (1980)) we obtain that $A^{*} \Phi_{d}=\lambda_{d} \Phi_{d}$. Now suppose that $\Phi_{d}$ is not strictly positive, i.e., there is a $\psi \in C_{+}, \psi \neq 0$ such that $\left\langle\Phi_{d}, \psi\right\rangle_{c}=0$. Then $\psi \in \mathscr{N}\left(\lambda_{d} I-A^{*}\right)^{\perp}=\mathscr{R}\left(\lambda_{d} I-A\right)$; hence $\lambda_{d} \phi-A \phi=\psi$ for some $\phi \in C$, hence

$$
\Delta\left(\lambda_{d}\right) \phi(0)=\psi(0)+H\left(\lambda_{d}\right) \psi+X_{+} \backslash\{0\} .
$$

A calculation very similar to the one performed in the proof of Theorem 5.4 of Heijmans (to appear, a) shows that

$$
\mathscr{R}\left(\Delta\left(\lambda_{d}\right)\right) \cap X_{+}=\{0\}
$$

and this is a contradiction. Therefore $\Phi_{d}$ is strictly positive.

An important question is whether or not the eigenvalue $\lambda_{d}$ is strictly dominant, i.e. $\operatorname{Re} \lambda<\lambda_{d}$ if $\lambda \in \sigma(A), \lambda \neq \lambda_{d}$. If Assumption 4.2 is satisfied, this would immediately imply that there exists a positive $\varepsilon$ such that $\operatorname{Re} \lambda<\lambda_{d}-\varepsilon$ if $\lambda \in \sigma(A), \lambda \neq \lambda_{d}$, because if this were not true then there would exist a sequence $\lambda_{n} \in \sigma(A)$ such that $\operatorname{Re} \lambda_{n}$ is strictly increasing and $\operatorname{Re} \lambda_{n} \rightarrow \lambda_{d}, n \rightarrow \infty$. But if $t>0$ is such that $T(t)$ is compact, then $e^{\lambda_{n} t} \in \sigma(T(t))$ and $\left|e^{\lambda_{n} t}\right|=e^{\operatorname{Re} \lambda_{n} \cdot t} \rightarrow e^{\lambda_{d} t}, n \rightarrow \infty$ which implies that $\sigma(T(t))$ has an accumulation point different from zero contradicting the compactness of $T(t)$.

The answer to the question concerning the strict dominance depends strongly on the dependence of the kernel $k_{\lambda}(x)$ of the operator $K(\lambda)$ on $\lambda$. As in Heijmans (to appear, a) we can prove the following result.

THEOREM 5.8. If Assumption 4.2 is satisfied, then there is an $\varepsilon>0$ such that $\operatorname{Re} \lambda<\lambda_{d}-\varepsilon$ if $\lambda \in \sigma(A) \backslash\left\{\lambda_{d}\right\}$.

If Assumption 4.2 is false for every $x \in\left[x_{0}, 1\right]$, then

$$
G\left(y^{-1}(\xi)\right)-G(\xi)=c, \quad \alpha \leqq \xi \leqq \beta,
$$

where $c$ is a constant, and we find that

$$
K(\lambda)=e^{-\lambda(r+c)} K(0),
$$

where the operator $K(0)$ does not depend on $\lambda$.

It follows immediately that in this case

$$
\lambda \in \sigma(A) \Rightarrow \lambda+k \cdot \frac{2 \pi i}{r+c} \in \sigma(A), \quad k \in \mathbb{Z},
$$


and Theorem 5.8 is certainly not true in this case. If Assumption 4.2 is fulfilled on a nonempty subset of $\left[x_{0}, 1\right]$ then the situation is more complicated but one can prove that Theorem 5.8 is still true (see Diekmann, Heijmans and Thieme (1985)).

As in Heijmans (to appear, a) it is possible to compute the so-called characteristic equation from which all eigenvalues of $A$ can be calculated in principle. Here we shall only do this for the special case $\beta<x_{0}$.

Let $\beta<x_{0}$ and let $v$ be a solution of

$$
K(\lambda) v=v .
$$

Then $v(x)$ is constant for $\beta \leqq x \leqq 1$ and we may take $v(x)=1, \beta \leqq x \leqq 1$. Then

$$
v(x)=\int_{\alpha}^{x} k_{\lambda}(\xi) v\left(y^{-1}(\xi)\right) d \xi=\int_{\alpha}^{x} k_{\lambda}(\xi) d \xi, \quad \alpha \leqq x<\beta .
$$

Since $v$ has to be continuous in $x=\beta$, we obtain

$$
1=\int_{\alpha}^{\beta} k_{\lambda}(\xi) d \xi
$$

and this equation determines the elements of $\sigma(A)$ if $\beta<x_{0}$.

6. The stable size distribution. Throughout this section we assume that Assumption 4.2 is satisfied. Let $\lambda_{d}$ be the strictly dominant eigenvalue of $A$, and let $\phi_{d}, \Phi_{d}$ be given by Theorem 5.7. Since $\lambda_{d}$ is a simple pole of $R(\lambda, A)$, we have the following decomposition of the state space $C$ (cf. Taylor and Lay (1980)):

$$
C=\mathscr{N}\left(\lambda_{d} I-A\right) \oplus \mathscr{R}\left(\lambda_{d} I-A\right),
$$

where $\mathscr{N}\left(\lambda_{d} I-A\right)$ is the one-dimensional space spanned by the positive eigenvector $\phi_{d}$. Let $P$ be the orthogonal projection on $\mathscr{N}\left(\lambda_{d} I-A\right)$ according to this decomposition; then $P$ is given by

$$
P \phi=\left\langle\Phi_{d}, \phi\right\rangle_{c} \cdot \phi_{d},
$$

where we have normalized $\Phi_{d}, \phi_{d}$ such that $\left\langle\Phi_{d}, \phi_{d}\right\rangle_{c}=1$. Let $\tilde{T}(t)$ be the restriction of $T(t)$ to $\mathscr{R}\left(\lambda_{d} I-A\right)$; then $r(\tilde{T}(t)) \leqq e^{\left(\lambda_{d^{-}}-\varepsilon\right) t}, t \geqq 0$, where we have used Theorem 5.8. A standard result from semigroup theory says that for all $0<\eta<\varepsilon$ there exits an $M(\eta) \geqq 1$ such that $\|\tilde{T}(t) \psi\| \leqq M(\eta) e^{\left(\lambda_{d}-\eta\right) t}\|\psi\|$, for all $\psi \in \mathscr{R}\left(\lambda_{d} I-A\right)$. Let $\phi \in C$; then $\phi=P \phi$ $+(I-P) \phi=\left\langle\Phi_{d}, \phi\right\rangle_{c} \cdot \phi_{d}+(I-P) \phi$ and therefore

$$
T(t) \phi=\left\langle\Phi_{d}, \phi\right\rangle_{c} \cdot e^{\lambda_{d} t} \cdot \phi_{d}+\tilde{T}(t)(I-P) \phi, \quad t \geqq 0
$$

and the following result is obtained.

THEOREM 6.1. For all $0<\eta<\epsilon$ there is a constant $M(\eta) \geqq 1$ such that for all $\phi \in C$

$$
\left\|T(t) \phi-\left\langle\Phi_{d}, \phi\right\rangle_{c} \cdot e^{\lambda_{d} t} \cdot \phi_{d}\right\| \leqq M(\eta) e^{\left(\lambda_{d}-\eta\right) t}\|\phi\|, \quad t \geqq 0 .
$$

For obvious reasons we call $\phi_{d}$ the stable size distribution.

Finally we mention that there is an alternative way to reach the main results exploiting the positivity of the semigroup. Using known results from positive semigroup theory (cf. Greiner (1981)), Theorem 5.8 follows immediately. The main problem is now to establish the algebraic simplicity of $\lambda_{d}$. This can be done by showing that the semigroup is not only positive but also irreducible (cf. Schaefer (1974), Greiner (1981)). However, the technical difficulties arising in this approach seem to be greater than in the one we have adopted. 
Acknowledgments. We gratefully acknowledge a number of valuable suggestions by Odo Diekmann and some stimulating discussions and correspondence with John Tyson on the biological aspects of the paper.

\section{REFERENCES}

G. I. Bell AND E. C. ANDerson (1967), Cell growth and division, I, A mathematical model with applications to cell volume distributions in mammalian suspension cultures, Biophys. J., 7, pp. 329-351.

O. Diekmann, H. J. A. M. Heimmans and H. R. Thieme (1984), On the stability of the cell size distribution, J. Math. Biology, 19, pp. 227-248.

(1985), On the stability of the cell size distribution, II Time periodic developmental rates, preprint.

H. R. Dowson (1978), Spectral Theory of Linear Operators, Academic Press, London-New York-San Francisco.

M. EISEN (1979), Mathematical models in Cell Biology and Cancer Chemotherapy, Lecture Notes in Biomathematics 30, Springer-Verlag, Berlin-Heidelberg-New York.

G. Greiner (1981), Zur Perron-Frobenius Theorie stark stetiger Halbgruppen, Math. Z., 177, pp. 401-423.

M. Gyllenberg (to appear), The size and scar distributions of the yeast Saccharomyces cerevisiae, J. Math. Biology.

J. K. Hale (1977), Theory of Functional Differential Equations, Springer-Verlag, Berlin-Heidelberg-New York.

K. B. HANNSGEN AND J. J. TYSON (1984), Stability of the steady-state size distribution in a model of cell growth and division, preprint.

H. J. A. M. Heimmans (to appear, a), An eigenvalue problem related to cell growth, J. Math. Anal. Appl.

(to appear, b), The dynamical behavior of the age-size distribution of a cell population, in Dynamics of Physiologically Structured Populations, J. A. J. Metz and O. Diekmann, eds., Lecture Notes in Biomathematics, Springer-Verlag, Berlin-Heidelberg-New York.

D. C. LAY (1970), Spectral analysis using ascent, descent, nullity and defect, Math. Ann., 184, pp. 197-214.

R. H. Martin, JR. (1976), Nonlinear Operators and Differential Equations in Banach Spaces, John Wiley, New York-London-Sydney-Toronto.

J. A. J. Metz and O. Diekmann (to appear), Dynamics of Physiologically Structured Populations, Lecture Notes in Biomathematics, Springer-Verlag, Berlin-Heidelberg-New York.

A. PAzy (1983), Semigroups of Linear Operators and Applications to Partial Differential Equations, Springer, New York.

H. H. SCHAEFER (1974), Banach Lattices and Positive Operators, Springer, New York.

J. W. Sinko AND W. STreifer (1971), A model for populations reproducing by fission, Ecology, 52, pp. 330-335.

A. E. TAYlor AND D. C. LAY (1980), Introduction to Functional Analysis, John Wiley, New York-Chichester-Brisbane-Toronto.

C. C. Travis and G. F. WebB (1974), Existence and stability for partial differential equations, Trans. Amer. Math. Soc., 200, pp. 395-418.

J. J. TYSON AND K. B. HANNSGEN (1984), The distribution of cell size and generation time in a model of the cell cycle incorporating size control and random transitions, preprint.

G. F. WebB (1985), Theory of Nonlinear Age-dependent Population Dynamics, Marcel Dekker, New York. 